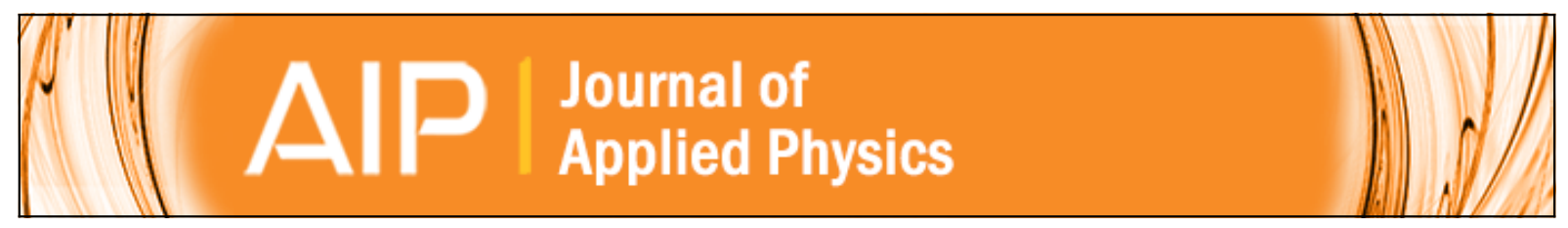

\title{
Polar orientation of a pendant anionic chromophore in thick layer-by-layer self-
} assembled polymeric films

Akhilesh Garg, Richey M. Davis, Cemil Durak, James R. Heflin, and Harry W. Gibson

Citation: Journal of Applied Physics 104, 053116 (2008); doi: 10.1063/1.2974094

View online: http://dx.doi.org/10.1063/1.2974094

View Table of Contents: http://scitation.aip.org/content/aip/journal/jap/104/5?ver=pdfcov

Published by the AIP Publishing

\section{Articles you may be interested in}

Layer-by-layer self-assembled conductor network composites in ionic polymer metal composite actuators with high strain response

Appl. Phys. Lett. 95, 023505 (2009); 10.1063/1.3179554

Coarse-grained molecular-dynamics simulations of the self-assembly of pentablock copolymers into micelles

J. Chem. Phys. 123, 234905 (2005); 10.1063/1.2137714

Ultrafast frequency-selective optical switching based on thin self-assembled organic chromophoric films with a large second-order nonlinear response

Appl. Phys. Lett. 81, 2169 (2002); 10.1063/1.1506413

Formation dynamics of layer-by-layer self-assembled films probed by second harmonic generation

J. Chem. Phys. 117, 3956 (2002); 10.1063/1.1495839

Optical properties of self-assembled thin-film of poly ( $p$-phenylene vinylene)s and its application to light-emitting devices with microring geometry

Appl. Phys. Lett. 76, 3227 (2000); 10.1063/1.126589

\section{MIT LINCOLN} LABORATORY CAREERS

Discover the satisfaction of innovation and service to the nation
- Space Control

- Air \& Missile Defense

- Communications Systems \& Cyber Security

- Intelligence, Surveillance and

Reconnaissance Systems
- Advanced
Electronics
- Tactical Systems
- Homeland
Protection
- Air Traffic Control

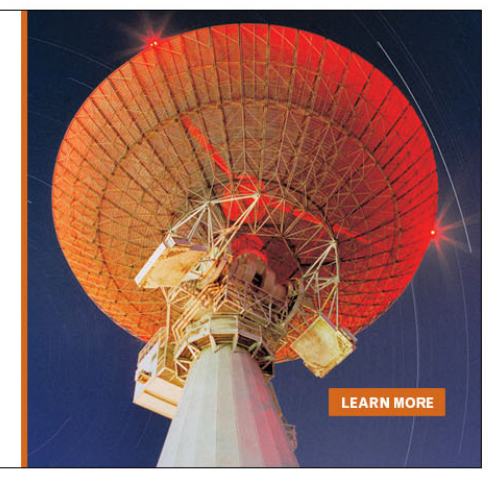




\title{
Polar orientation of a pendant anionic chromophore in thick layer-by-layer self-assembled polymeric films
}

\author{
Akhilesh Garg, ${ }^{1}$ Richey M. Davis, ${ }^{1, a)}$ Cemil Durak, ${ }^{2}$ James R. Heflin, ${ }^{2}$ and \\ Harry W. Gibson ${ }^{3}$ \\ ${ }^{1}$ Department of Chemical Engineering, Virginia Tech, Blacksburg, Virginia 24061, USA \\ ${ }^{2}$ Department of Physics, Virginia Tech, Blacksburg, Virginia 24061, USA \\ ${ }^{3}$ Department of Chemistry, Virginia Tech, Blacksburg, Virginia 24061, USA
}

(Received 7 January 2008; accepted 30 June 2008; published online 9 September 2008)

\begin{abstract}
Multilayer films with up to 600 bilayers and $740 \mathrm{~nm}$ thickness were fabricated using the alternating

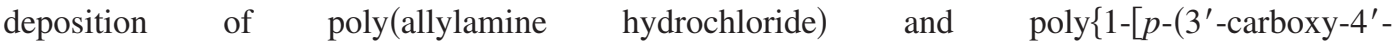
hydroxyphenylazo)benzenesulfonamido]-1,2-ethandiyl\} on glass substrates. Linear relationships for absorbance, thickness, and the square root of the second harmonic intensity versus the number of bilayers demonstrates that the films have long range polar order and optical homogeneity. The deposition conditions (i.e., $p \mathrm{H}$ of the solutions, solvent quality, deposition and rinsing times) are critical variables in fabricating layer-by-layer thick films that exhibit long range polar orientation of chromophores. The ability to fabricate noncentrosymmetric films on the order of a micron thick with bulk second order nonlinear optical responses is crucial because it enables the development of nonlinear optical waveguide devices such as electro-optic modulators. (C) 2008 American Institute of Physics. [DOI: 10.1063/1.2974094]
\end{abstract}

\section{INTRODUCTION}

The alternate adsorption of polycations and polyanions on a charged surface leads to the formation of ionic selfassembled multilayer (ISAM) films, also commonly referred to as layer-by-layer (LbL) films. When a polyelectrolyte adsorbs onto an oppositely charged surface, charge reversal on the surface typically occurs; this facilitates the adsorption of the next layer of oppositely charged polymer. In principle, films with an arbitrary number of bilayers can be fabricated in this fashion. This method, developed by Decher and Hong, ${ }^{1}$ has many potential applications in fields such as integrated molecular optics, electronic devices, and biosensors. ${ }^{2}$ Films made in this way can exhibit second order nonlinear optical (NLO) effects if they satisfy the criterion that one of the constituents contains a chromophore that can be oriented in a polar manner. ${ }^{3,4}$ Films exhibiting second order NLO effects have applications in electro-optic (EO) modulators, which convert electrical signal to optical signal and thus play an important role in today's communication network.

For noncentrosymmetric organic films, the second order nonlinear susceptibility, $\chi^{(2)}$ is directly proportional to the chromophore density and hyperpolarizability as given by the equation

$$
\chi^{(2)}=N F \beta\left\langle\cos ^{3} \bar{\psi}\right\rangle,
$$

where $N$ is the chromophore density, $F$ is the local field factor, $\beta$ is the hyperpolarizability, and $\bar{\psi}$ is the orientation angle of the chromophores with respect to the surface normal. Rational design thus involves choosing a chromophore

\footnotetext{
a) Author to whom correspondence should be addressed. Electronic mail: rmdavis@vt.edu.
}

with a suitably high $\beta$ and incorporating it into a film with high density $N$, and low tilt angle $\bar{\psi}$.

There are several requirements for a practical, organic, EO modulator film, including (1) the absorbance of the chromophores should be minimal in the wavelength range in which it operates, (2) film $\chi^{(2)}$ should exhibit no significant loss upon heating needed for device integration; typically a long term thermal stability for operating temperatures as high as $125^{\circ} \mathrm{C}$ and short term stability for temperatures approaching $200{ }^{\circ} \mathrm{C},{ }^{5}$ (3) the film thickness needs to be $\geq 1 \mu \mathrm{m}$ in order to sustain waveguiding at wavelengths typically used in optical fibers for telecommunications, (4) polar ordering of the chromophores should be maintained over this film thickness, and (5) films should be compositionally and hence optically homogeneous to minimize losses due to scattering. In this paper, we work with films that satisfy criteria (1) and (2) and focus on requirements (3)-(5) with films of thicknesses up to $740 \mathrm{~nm}$. This is a substantial increase over the $120 \mathrm{~nm}$ thickness for the previously reported thickest films that exhibited proper scaling of thickness and NLO response $^{6}$ and indicates the suitability of ISAM films for EO waveguide devices.

Approaches for fabricating organic films for EO modulator applications include guest/host poled polymeric materials, which have been shown to exhibit high $\chi^{(2)}{ }^{7}$ In the guest/host poled polymer method, the chromophores are heated above the glass transition temperature and an electric field is applied; this leads to chromophore orientation. The chromophores are frozen in this position when the temperature of the system is rapidly brought down to room temperature. While the eventual randomization of the orientation back to the isotropic state has proven a challenging problem, advances continue to be made through the use of higher $T_{g}$ hosts, covalent attachment of the chromophore to the poly- 
mer, cross-linked polymers, and dendrimeric structures. ${ }^{8-12}$ Using supramolecular self-assembled molecular glasses, an EO coefficient as high as $327 \mathrm{pm} / \mathrm{V}$ was achieved, approximately ten times higher than $\mathrm{LiNbO}_{3}$, and showed $83 \%$ temporal alignment stability for 3 months at room temperature. ${ }^{13}$ Noncentrosymmetric Langmuir Blodgett (LB) films with $\chi^{(2)}$ values as large as $760 \times 10^{-9}$ esu have been fabricated, approximately four times larger than that of $\mathrm{LiNbO}_{3} .{ }^{14} \mathrm{How}-$ ever, LB films tend to possess poor mechanical and thermal stabilities owing to the relatively weak van der Waals interactions between layers. Alternatively, several variations have been developed for growth of polar, self-assembled multilayers using siloxane chemistry. A recent procedure can deposit $1 \mathrm{ML}$ (monolayer) in 40 min with $\chi^{(2)}=430 \times 10^{-9}$ esu. $^{15,16}$ A related approach involving sol-gel chemistry resulted in ternary films consisting of alternating layers of $\mathrm{ZrO}_{2}$, carboxyl-functionalized azobenzene dyes, and poly(diallydimethylammonium chloride), which led to $\chi_{z z z}^{(2)}$ values of $(222-899) \times 10^{-9}$ esu. The processing time for a ternary layer deposition cycle was approximately $25 \mathrm{~min}$ and film thicknesses of up to $140 \mathrm{~nm}$ were reported. ${ }^{17}$

For materials exhibiting NLO effects, polar ordering of the chromophores can be studied using second harmonic generation (SHG) measurements. The second harmonic intensity should increase quadratically with the film thickness if polar order is maintained and the film thickness is much less than the coherence length (typically on the order of $10 \mu \mathrm{m}$.). For LbL films formed via electrostatic and hydrogen bonding effects, polar ordering depends on several factors including the chemistry and architecture of the component polymers. In one study, complexation between a cationic cyclodextrin derivative and a polyanion with an azobenzene side group led to quadratic growth of SHG for films up to 30 bilayers thick but not beyond that. ${ }^{18}$ Nonquadratic growth of SHG was found for films consisting of up to ten bilayers made with a cationic polyionene containing an azobenzene side group and poly(vinyl sulfate). ${ }^{19}$ Similar results were reported for films consisting of up to six bilayers made with a quaternary polyamine and the anionic azobenzene-containing polymer that is the focus of the present work. ${ }^{20}$ Another study showed a linear increase in absorbance up to at least 80 bilayers; however, the SHG failed to show quadratic scaling above 50 bilayers. $^{21}$ The effect of polycation type-branched poly(ethyleneimine) and linear poly(diallyldimethylammonium chloride) —on the orientation of the azo group in polycation/poly\{1[ $p$-(3'-carboxy-4'-hydroxyphenylazo)benzenesulfonamido]1,2-ethandiyl\} (PCBS) LbL films was studied using absorbance measurements although NLO effects were not probed. ${ }^{22}$ A lyotropic, cationic polyionene and strong polyanions were used to make highly ordered films consisting of up to 50 bilayers, but SHG was not studied. ${ }^{23}$ In most of these studies where film thickness was measured, it was found that the film thickness and chromophore content- the latter characterized by absorbance measurements-scaled linearly with the bilayer number. While this linear scaling is not required for chromophore ordering that gives rise to significant values of $\chi^{(2)}$, linear scaling is necessary to yield films with a uniform value of $\chi^{(2)}$ throughout the film, which would be desirable for an optical waveguide in an EO modulator. Thus, one of the issues that we explore in this present work is the set of processing conditions that gives rise to linear growth in film thickness and chromophore concentration, as well as quadratic scaling of the SHG intensity with the film thickness.

Prior to the present work, the only studies involving LbL film deposition that showed long range polar ordering at thicknesses greater than $100 \mathrm{~nm}$ were those by Kang et al. and by Heflin et al.; ${ }^{6}$ the latter involved films made by the ISAM process using a polyanion with an azobenzene side group and poly(allylamine hydrochloride) (PAH). A very interesting feature of these ISAM films is their excellent thermal and temporal stability, an important factor for EO modulator applications and one that has been a significant limitation for commercialization of organic EO modulator materials. In the previous work by Heflin co-workers, ${ }^{6,24}$ ISAM films made with PAH and an azobenzene-containing polyanion, PS-119, exhibited complete recovery in $\chi^{(2)}$ after the films were heated at $150{ }^{\circ} \mathrm{C}$ for $15 \mathrm{~h}$ and then cooled to room temperature. These films have shown no measurable loss in $\chi^{(2)}$ upon storage under atmospheric conditions at room temperature for 10 years. Although the temporal and thermal stability measurements were not performed on the films fabricated in this study using PAH/PCBS, the similarity between PCBS and PS-119 suggests that the films fabricated using PCBS may show similar levels of thermal and temporal stability. However, this is yet to be verified and is a subject of a future paper. Finally, films fabricated using a hybrid covalent/ionic self-assembled technique have also exhibited excellent thermal stability at temperatures as high as $150{ }^{\circ} \mathrm{C}$ for $24 \mathrm{~h}$ and temporal stability for over 14 months under ambient conditions. ${ }^{25}$ The sample maintained $90 \%$ of the SHG signal upon heating for $24 \mathrm{~h}$ at $150{ }^{\circ} \mathrm{C}$ and completely recovered after cooling to room temperature; the change at elevated temperature was believed to be due to trans-cis isomerization of the azobenzene chromophore.

The main focus of the present work is a study of the processing conditions-deposition and rinsing times and solution $p \mathrm{H}$ values which affect the degree of ionization of the polyelectrolytes - necessary to fabricate organic ISAM films with the requisite long range polar ordering at thicknesses approaching the $1 \mu \mathrm{m}$ limit and with the necessary optical homogeneity.

\section{EXPERIMENTAL}

\section{A. Materials}

PAH [Fig. 1(a)] was used as the polycation $\left(M_{w}\right.$ $\sim 70 \mathrm{kDa}$; Aldrich). PCBS [Fig. 1(b)] (Aldrich) was used as the polyanionic chromophore. De-ionized (DI) water (Barnstead ROpureST; model D6311) with a resistivity of over $17 \mathrm{M} \Omega \mathrm{cm}$ was used. PAH was chosen as a polycation because it has shown good film quality and scaling of the SHG intensity, in contrast to other polycations, in our prior work on ISAM NLO films. PCBS has an azobenzene pendant group that has a sufficiently high hyperpolarizability, $\beta$, to give a substantial SHG signal. Microscope slides (Fisher Scientific) frosted on the end of one side were used as sub- 


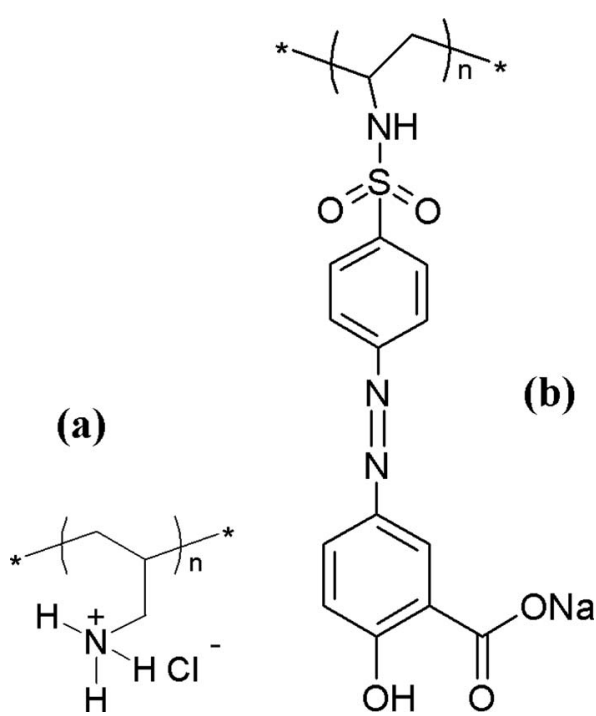

FIG. 1. Structures of (a) PAH and, (b) PCBS.

strates. Ammonium hydroxide (28\%-30\%, Aldrich), hydrogen chloride (35.5\%-38\%, EM Science), and hydrogen peroxide $(31.8 \%$, Fisher) were used for cleaning the glass substrate using the procedure described below.

\section{B. Fabrication of slides}

To obtain films with good compositional and optical homogeneity, the substrates were cleaned carefully using the RCA cleaning procedure. ${ }^{26}$ The slides were immersed in a solution of 6:2:1 $\mathrm{H}_{2} \mathrm{O}, \mathrm{H}_{2} \mathrm{O}_{2}$, and $\mathrm{NH}_{4} \mathrm{OH}$ at $70{ }^{\circ} \mathrm{C}$ for 20 min, rinsed carefully with DI water, immersed in a fresh solution of $6: 2: 1 \mathrm{H}_{2} \mathrm{O}, \mathrm{H}_{2} \mathrm{O}_{2}, \mathrm{HCl}$ at $70^{\circ} \mathrm{C}$ for $20 \mathrm{~min}$, rinsed again with DI water and baked at $130{ }^{\circ} \mathrm{C}$ for at least an hour.

A first set of the films were made using a StratoSequence $^{\mathrm{TM}}$ Mark VI robotic deposition system (nanoStrata Inc.). It consists of eight beakers on a rotating platform that is raised and lowered by nitrogen gas pressure controlled by a computer. The polyelectrolytes (PAH and PCBS) and the rinse water were placed in $150 \mathrm{ml}$ beakers. Four slides were held in a fixture that can either be stationary or can be made to rotate when immersed in the beakers. To minimize convection and diffusion transport effects, the slide fixture was always rotated at $60 \mathrm{rpm}$. An effective Reynolds number of 320 was estimated using the width of the slide as the characteristic length. There were two separate dipping cycles used: (1) slides were dipped in a polyelectrolyte solution for 2 min followed by three rinsing steps using DI water, each lasting $45 \mathrm{~s}$ and (2) slides were dipped in a polyelectrolyte solution for $45 \mathrm{~s}$ followed by one rinsing step using DI water lasting $45 \mathrm{~s}$. In both dipping cycles, the rinse water was replaced after every rinsing cycle whereas the polyelectrolyte solution was replaced after every $6 \mathrm{~h}$ to avoid dilution.

Another set of the films were fabricated using a Richard-Allen Scientific DS/50 automated slide stainer. The slide stainer was equipped with 20 polyphenyline sulfide (PPS) Ryton static deposition chambers, one rinse bath (also constructed of PPS) and a drying chamber. An aquarium powerhead pump was added to the active-rinse bath to in- crease fluid circulation and thus rinsing efficiencies. No convection was used in the deposition baths. The films were deposited onto glass microscope slides (Fisher Scientific) prepared as noted above. For all films made with the DS/50 slide stainer, the polymer deposition and water rinse times were $2 \mathrm{~min}$.

For all experiments, the concentrations of the PAH and PCBS solutions were $10 \mathrm{mM}$ on a repeat unit basis. The repeat unit molecular weight of PAH is $93.56 \mathrm{~g} / \mathrm{mol}$ and of PCBS is $369.3 \mathrm{~g} / \mathrm{mol}$; the concentration (in $\mathrm{mg} / \mathrm{ml}$ ) of PAH was $0.936 \mathrm{mg} / \mathrm{ml}$ and for PCBS was $3.693 \mathrm{mg} / \mathrm{ml}$. The $p \mathrm{Hs}$ of the polymer solutions were adjusted using $1.0 \mathrm{M}$ or $0.1 \mathrm{M}$ solutions of sodium hydroxide and hydrogen chloride. The $p \mathrm{H}$ of the solutions drifted no more than $\pm 0.1 p \mathrm{H}$ units during the course of the experiment.

\section{Film characterization}

Absorbance measurements were done using a Perkin Elmer Lambda $25 \mathrm{UV} /$ visible system. The films exhibited an absorbance maximum, $\lambda_{\max }$, at $360 \mathrm{~nm}$. At $360 \mathrm{~nm}$, the absorbance values of the thicker films surpassed the maximum absorbance value of 6.63 that the instrument can measure and therefore the measurements were taken at $450 \mathrm{~nm}$ wavelength. Film thicknesses were measured using a variable angle spectroscopic ellipsometer (J. A. Woolam ellipsometer VB-200 with WVASE32 software version 3.361). The ellipsometric analysis was done in the vicinity of the Brewster angle because ellipsometry measurements are most sensitive to film characteristics in the vicinity of the Brewster angle. ${ }^{27,28}$ An initial scan was done for wavelengths from 300 to $800 \mathrm{~nm}$ at $50 \mathrm{~nm}$ intervals and this wavelength range was then repeated over angles from $50^{\circ}-70^{\circ}$ at $4^{\circ}$ intervals to find the Brewster angle which, for the PAH/PCBS system was found to be $62^{\circ}$. Following the determination of the Brewster angle, the sample was scanned at wavelengths from 300 to $800 \mathrm{~nm}$ at $10 \mathrm{~nm}$ intervals in the vicinity $\left( \pm 2^{\circ}\right)$ of the Brewster angle in $1^{\circ}$ increments. The sample was scanned at three different positions to check for the film homogeneity. The experimental data collected this way were then fitted with one Lorentz oscillator where the wavelength of the absorbance peak was fixed at the value of $360 \mathrm{~nm}$ as measured by UV-visible spectroscopy. Atomic force microscopy (AFM) pictures were taken using a Veeco Dimension 3100 Nanoman AFM. A representative area of $20 \times 20 \mu \mathrm{m}^{2}$ was selected on each slide fabricated at different deposition conditions to study the morphology of these films.

SHG measurements were used to calculate the second order susceptibility, $\chi^{(2)}$. A linearly polarized Nd:YAG (yttrium aluminum garnet) fundamental beam with the wavelength of $1064 \mathrm{~nm}$, a pulse width of $10 \mathrm{~ns}$, and a pulse energy of $\sim 1 \mathrm{~mJ}$ was used. The second harmonic intensity was measured as a function of the incident angle. The incident angle/intensity data were used to calculate the $\chi^{(2)}$ parameter. Figure 2 shows a representative plot of angle versus intensity for the PAH/PCBS films.

The effective second order susceptibility $\chi_{\text {eff }}^{(2)}$ was calculated using 


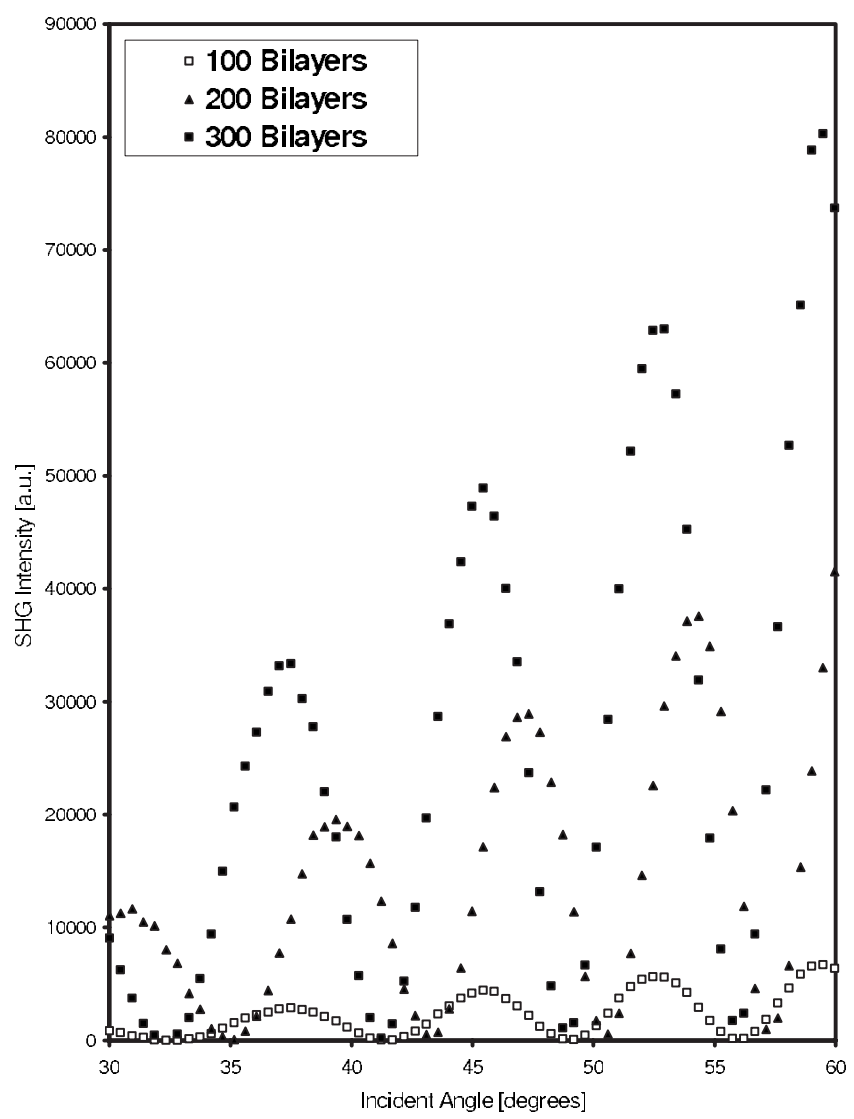

FIG. 2. SHG Intensity vs angle of incidence for 100, 200. and 300 bilayer $\mathrm{PAH} / \mathrm{PCBS}$ films. The deposition conditions were: $\mathrm{PAH}: 10 \mathrm{mM}, p \mathrm{H}=7.0,2$ min deposition time and $2 \mathrm{~min} 15 \mathrm{~s}$ rinse time; same for PCBS. The experiments were carried out at room temperature. Typical error bars are smaller than the size of the data points.

$$
\chi_{\text {eff }}^{(2)}=\chi_{\text {ref }}^{(2)}\left(\frac{l_{\text {ref }}}{l_{s, \text { bilayer }}}\right)\left(\frac{m}{\sqrt{I_{\text {ref }}^{2 \omega}}}\right),
$$

where $\chi_{\text {ref }}^{(2)}$ is the second order susceptibility of the reference material, $1.5 \times 10^{-9}$ esu, $l_{\text {ref }}$ is the thickness of the reference material, $75.3 \mathrm{~nm}, m$ is the slope of square root of SHG intensity versus number of bilayers, $I_{\mathrm{ref}}^{2 \omega}$ is the intensity of the reference material and $l_{s \text {,bilayer }}$ is the thickness per bilayer obtained from ellipsometric measurements.

The reference used was a 68 bilayer PS-119/PAH ISAM film that has had a constant value of thickness and second order susceptibility over the past 10 years. ${ }^{6}$

For a $p$-polarized light at a given incident angle $\theta, \chi_{\text {eff }}^{(2)}$ is related to the $\chi^{(2)}$ tensor components $\chi_{z z z}^{(2)}$ and $\chi_{z x x}^{(2)}$ by

$$
\chi_{\text {eff }}^{(2)}=3 \chi_{z x x}^{(2)} \sin \theta \cos ^{2} \theta+\chi_{z z z}^{(2)} \sin ^{3} \theta .
$$

The tilt angle was determined by comparing the SHG intensity generated for incident $s$ - and $p$-polarizations of the light. The maximum harmonic intensity generated from $p$-polarized light is represented by $I_{2 \omega}^{p \rightarrow p}$ and the maximum harmonic intensity generated from $s$-polarized light is represented by $I_{2 \omega}^{S \rightarrow p} . I_{2 \omega}^{p \rightarrow p}$ and $I_{2 \omega}^{S \rightarrow p}$ are related to the ratio of $\chi_{\mathrm{eff}}^{(2)}$ tensor components $\chi_{z z z}^{(2)}$ and $\chi_{z x x}^{(2)}$ by

\begin{tabular}{|c|c|c|c|c|}
\hline Deposition Condition & $\begin{array}{c}\mathrm{PAH} \\
\text { solution } \\
p \mathrm{H}\end{array}$ & $\begin{array}{c}\text { PCBS } \\
\text { solution } \\
p \mathrm{H}\end{array}$ & $\begin{array}{c}\text { Deposition } \\
\text { time } \\
\text { (min:s) }\end{array}$ & $\begin{array}{l}\text { Rinse } \\
\text { Time } \\
\text { (min:s) }\end{array}$ \\
\hline (1) $(\mathrm{PAH}-9 / \mathrm{PCBS}-8)_{2 / 2} \min -15 \mathrm{~s}$ & 9.0 & 8.0 & 2:00 & $2: 15$ \\
\hline (2) $(\mathrm{PAH}-7 / \mathrm{PCBS}-7)_{2 / 2} \min -15 \mathrm{~s}$ & 7.0 & 7.0 & 2:00 & $2: 15$ \\
\hline (3) $(\mathrm{PAH}-7 / \mathrm{PCBS}-7)_{45 / 45 \mathrm{~s}}$ & 7.0 & 7.0 & $0: 45$ & $0: 45$ \\
\hline
\end{tabular}

TABLE I. Different deposition conditions for LbL experiments.

$$
\frac{\chi_{z z z}^{(2)}}{\chi_{z x x}^{(2)}}=\sqrt{\frac{I_{2 \omega}^{p \rightarrow p}}{I_{2 \omega}^{s \rightarrow p}}} \csc ^{2} \theta-3 \cot ^{2} \theta .
$$

The tilt angle is related to the ratio of tensor components by the equation

$$
2 \cot ^{2} \bar{\psi}=\frac{\chi_{z z z}^{(2)}}{\chi_{z x x}^{(2)}}
$$

Using Eqs. (2)-(5), the tilt angle and $\chi_{z z z}^{(2)}$ component of the $\chi_{\text {eff }}^{(2)}$ tensor were calculated. All film measurements were made after drying the films in air under ambient conditions.

\section{RESULTS AND DISCUSSION}

A series of different bilayer number films were fabricated by depositing PAH and PCBS under three different conditions. The first deposition condition was PAH $10 \mathrm{mM}$, $p \mathrm{H}=9.0$; PCBS $10 \mathrm{mM}, p \mathrm{H} 8.0$ with 2 min deposition time for both PAH and PCBS, and a total rinse time of $2 \min 15 \mathrm{~s}$. This is abbreviated as (PAH-9/PCBS-8) $2 / 2$ min-15 s: the number after the polymer gives the $p \mathrm{H}$ at which it was deposited and subscripts define the polymer deposition time followed by the rinsing time. This was chosen to probe the effect of ionization of PAH on film properties because changing the $p \mathrm{H}$ changes the degree of ionization of PAH. The second deposition condition was PAH and PCBS both $10 \mathrm{mM}, p \mathrm{H}$ $=7.0$ with deposition time of $2 \mathrm{~min}$ and rinsing time of $2 \mathrm{~min}$ $15 \mathrm{~s}$ for both polymers, written in short form as (PAH-7/PCBS-7) $2 / 2$ min-15 s. The third deposition condition was PAH and PCBS both $10 \mathrm{mM}, p \mathrm{H}=7.0$ with deposition time of $45 \mathrm{~s}$ and rinsing time of $45 \mathrm{~s}$ for both polymers, written in short form as (PAH-7/PCBS-7 $)_{45 / 45} \mathrm{~s}$. The last two conditions were chosen to probe the effect of deposition and rinsing time on ISAM film properties. These conditions are summarized in Table I.

The deposition conditions (1) and (2) were used to compare the effect of $p \mathrm{H}$-induced ionization of PAH while deposition and rinsing times were maintained constant. Deposition conditions (2) and (3) were used to study the effect of deposition and rinsing time on properties of films made at the same $p \mathrm{H}$ conditions.

\section{A. Effect of deposition and rinsing times}

The effects of deposition and rinsing times were studied for the conditions (PAH-7/PCBS-7 $)_{2 / 2} \min -15 \mathrm{~s}$ and (PAH-7/PCBS-7 $)_{45 / 45}$ s; the concentration and $p \mathrm{H}$ were kept the same. For (PAH-7/PCBS-7 $)_{2 / 2}$ min-15 s and (PAH-9/PCBS-8) $)_{2 / 2}$ min-15 s, 500 bilayers were deposited on 


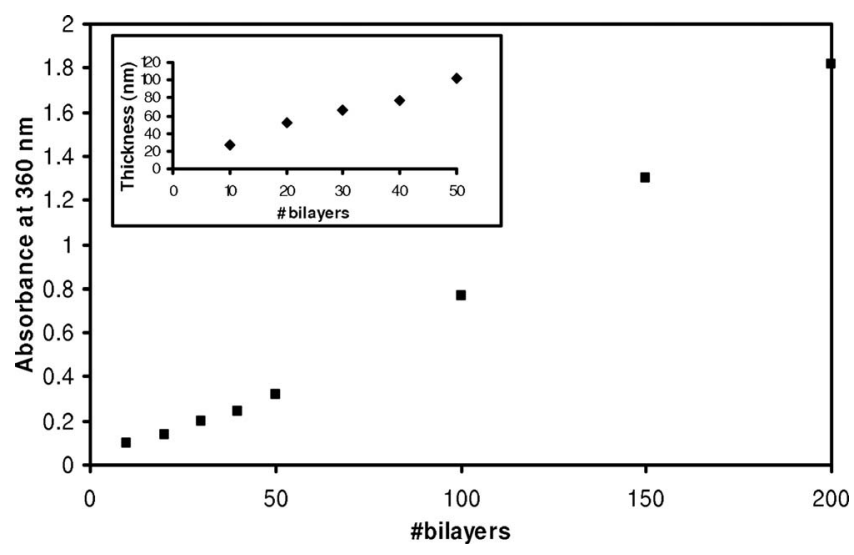

FIG. 3. Absorbance at $360 \mathrm{~nm}$ vs number of bilayers on a single side for (PAH-7/PCBS-7) $45 / 45$ s. The concentrations of PAH and PCBS were 10 $\mathrm{mM}$. The inset shows the thickness of these films up to 50 bilayers on a single side.

each side of the slide, whereas for the condition $(\mathrm{PAH}-7 / \mathrm{PCBS}-7)_{45 / 45 \mathrm{~s}}, 200$ bilayers were deposited on each side of the slide.

As shown in Fig. 3, absorbance measurements for the deposition condition (PAH-7/PCBS-7 $)_{45 / 45 \mathrm{~s}}$ taken at 360 $\mathrm{nm}$ show a linear increase up to 50 bilayers; however, when more bilayers are deposited, a nonlinear increase in absorbance is observed, indicating a nonlinear growth in these films. This could be because of insufficient rinsing times to remove all nonelectrostatically adsorbed polyelectrolyte and carry over of contaminants from rinsing baths to the next polyelectrolyte solution; this can lead to higher adsorption of next layer and over subsequent bilayers would result in an exponential growth. The inset shows the thickness versus the number of bilayers as measured by ellipsometry. Above 50 bilayers, thickness data could not be obtained because of low film quality which is noted in Table II in terms of high standard deviations observed at higher bilayer number. The standard deviations for the deposition conditions (PAH-7/PCBS-7) $2 / 2$ min-15 s and (PAH-9/ PCBS-8) $2 / 2$ min-15 $\mathrm{s}$ in Table II are discussed later in the paper.

Film morphology as embodied by the bilayer thickness and the mode of growth-linear versus exponential change in thickness with increasing bilayer number-should also play a role in long range polar ordering of NLO chromophores. This has not been studied in detail in previous work, but some related, recent studies are relevant here. Non-

TABLE II. Percent standard deviation of ${ }_{\sqrt{S H G}}$ to characterize optical homogeneity of films at different deposition conditions.

\begin{tabular}{|c|c|c|}
\hline Deposition condition & Bilayer number & $\sigma_{\nabla_{\mathrm{SHG}}}$ \\
\hline \multirow[t]{2}{*}{$(\mathrm{PAH}-7 / \mathrm{PCBS}-7)_{45 / 45 \mathrm{~s}}$} & 150 & 14.1 \\
\hline & 200 & 43.4 \\
\hline \multirow[t]{3}{*}{$(\mathrm{PAH}-7 / \mathrm{PCBS}-7)_{2 / 2} \min -15 \mathrm{~s}$} & 200 & 6.1 \\
\hline & 400 & 9.3 \\
\hline & 500 & 6.2 \\
\hline \multirow[t]{3}{*}{$(\mathrm{PAH}-9 / \mathrm{PCBS}-8)_{2 / 2} \min -15 \mathrm{~s}$} & 200 & 5.2 \\
\hline & 400 & 17.5 \\
\hline & 500 & 27.3 \\
\hline
\end{tabular}

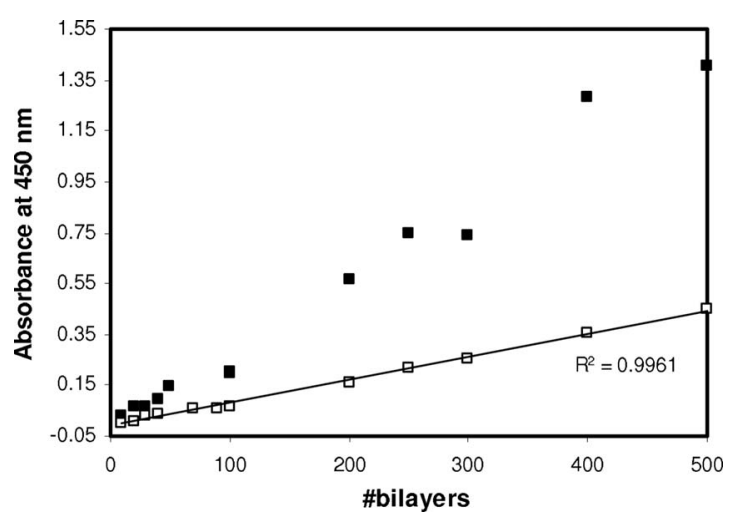

FIG. 4. Absorbance vs number of bilayers for the slide series fabricated using PAH/PCBS at two different deposition conditions (PAH-7/PCBS-7) $2 / 2$ min-15 s [open squares] and (PAH-9/PCBS-8) $2 / 2$ min-15 s [filled squares]. The concentrations of PAH and PCBS were $10 \mathrm{mM}$ for all deposition conditions.

linear growth of thickness and absorbance with increasing bilayer number was found for ISAM films comprised of polyelectrolytes with low charge densities, $\sim 10 \mathrm{~mol} \%$ ionic functionality. ${ }^{29}$ In a related study, reducing the solvent quality by using water/ethanol mixtures and by adding $\mathrm{NaCl}$ resulted in progressively thicker films and exponential growth in thickness and mass loading with increasing bilayer number. $^{30}$

\section{B. Effect of $p H$}

The effect of the polymer solution $p \mathrm{H}$ on film properties was studied for the conditions (PAH-9/PCBS-8) $2 / 2$ min-15 s and (PAH-7/PCBS-7) $2 / 2$ min-15 s: the concentration of the polyelectrolytes and the deposition and rinsing times were kept constant. The effect of $p \mathrm{H}$ on the structure of multilayer ISAM films has been extensively studied. ${ }^{31-33} \mathrm{PAH}$ has a $p K_{a}$ of $8.7^{34}$ and thus depositing it at $p \mathrm{H}>p K_{a}$ results in relatively thick layers with loops and tails because of the reduced charge repulsion on the polymer, which would, in turn, lead to increased adsorption of PCBS. In a separate paper, the effect of $p \mathrm{H}$ on bilayer thickness and film composition for hydrated films comprised of PAH and PCBS has been studied using a combination of quartz crystal microbalance with dissipation monitoring (QCM-D), ellipsometry, and absorbance measurements. ${ }^{35}$

Film quality was assessed with measurements of absorbance, thickness, AFM, and SHG. The films fabricated at the condition (PAH-7/PCBS-7) $2 / 2$ min-15 s exhibited excellent optical homogeneity up to 500 bilayers, the maximum number deposited, whereas films fabricated using the deposition condition (PAH-9/PCBS-8) $2 / 2$ min-15 s showed good optical homogeneity up to 300 bilayers. Figure 4 shows the absorbance versus bilayer number up to 500 bilayers (each side) for the two series fabricated using different deposition conditions. Although the peak of the absorbance spectrum is at $360 \mathrm{~nm}$, the absorbance values were taken at $450 \mathrm{~nm}$ for the deposition conditions: (PAH-9/PCBS-8) $2 / 2$ min-15 s and (PAH-7/PCBS-7) $2 / 2$ min-15 s because at $360 \mathrm{~nm}$ the absorbance value surpassed the maximum limit of the spectrophotometer. The absorbance per bilayer at $450 \mathrm{~nm}$ for 


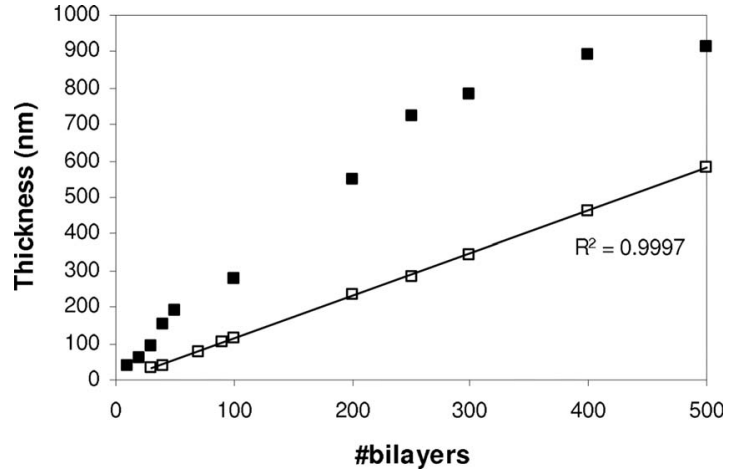

FIG. 5. Thickness vs number of bilayers for (PAH-7/PCBS-7) $2 / 2$ min-15 s [open squares] and (PAH-9/PCBS-8) $2 / 2$ min-15 s [filled squares]. The thickness obtained per bilayer was $1.17 \pm 0.01 \mathrm{~nm}$ for the first deposition condition and $2.75 \pm 0.09 \mathrm{~nm}$ for the second deposition condition up to $250 \mathrm{bi}$ layers. The concentrations of PAH and PCBS were $10 \mathrm{mM}$ for all deposition conditions.

$(\mathrm{PAH}-7 / \mathrm{PCBS}-7)_{2 / 2}$ min-15 s was $9.0( \pm 2 \%) \times 10^{-4}$ and for $(\mathrm{PAH}-9 / \mathrm{PCBS}-8)_{2 / 2}$ min-15 s was $2.9( \pm 5 \%) \times 10^{-3}$ up to 500 bilayers for both conditions.

Thickness data for the deposition conditions: (PAH-9/PCBS-8) $2 / 2$ min-15 s and (PAH-7/PCBS-7) $2 / 2$ min-15 s are shown in Fig. 5. The ellipsometric measurements show that the thickness of PAH/PCBS films increases linearly with number of bilayers for deposition condition (PAH-7/PCBS-7) 2/2 min-15 s whereas the thickness increases roughly linearly up to 250 bilayers for the deposition condition (PAH-9/PCBS-8) $2 / 2$ min-15 s. The thickness per bilayer for $(\mathrm{PAH}-7 / \mathrm{PCBS}-7)_{2 / 2}$ min-15 s was $1.17( \pm 0.01) \mathrm{nm}$ while the thickness per bilayer for (PAH-9/ PCBS-8) $2 / 2$ min-15 s was 2.75 ( \pm 0.09$)$ nm up to 250 bilayers. This more than twofold increase in bilayer thickness when the $\mathrm{PAH}$ was deposited at $p \mathrm{H} 9$ was due to the reduced charge density of the chains which leads to thicker monolayers. These, in turn, provide for more adsorption sites for PCBS chains when deposited at $p \mathrm{H} \mathrm{8}$, leading to thicker bilayers. This is consistent with the observation of Barrett et $a{ }^{31}$ in which they deposited PAH and PCBS at different $p \mathrm{H}$ conditions and observed that the bilayer thickness increased by approximately sixfold and the optical absorbance increased by a factor of 20 as the $p \mathrm{H}$ of the deposition solutions varied from 3 to 10 . Molecular control over the formation of ISAM films can thus be achieved by manipulating the $p \mathrm{H}$ of the weak polyelectrolyte. ${ }^{32}$ Using PAH and poly (acrylic acid) (PAA), bilayers with thicknesses ranging from less than 1-12 $\mathrm{nm}$ have been prepared at different $p \mathrm{H}$ conditions. $^{36}$ For the films made with the (PAH-9/PCBS-8) 2/2 min-15 s deposition conditions for bilayer number $>250$, growth in film thickness changed to a sublinear manner while the plot of absorbance versus bilayer number in Fig. 4 did not. This implies that the PCBS content of the films in this region was higher than for films with bilayer number $<250$ since the higher PCBS content would compensate for the thinner bilayers. This loss of linearity correlated with the appearance of optical inhomogeneities for bilayer number $>250$ and the loss of long range polar ordering, as shown in Fig. 5. These optical defects are scattering centers which most likely arose from compositional

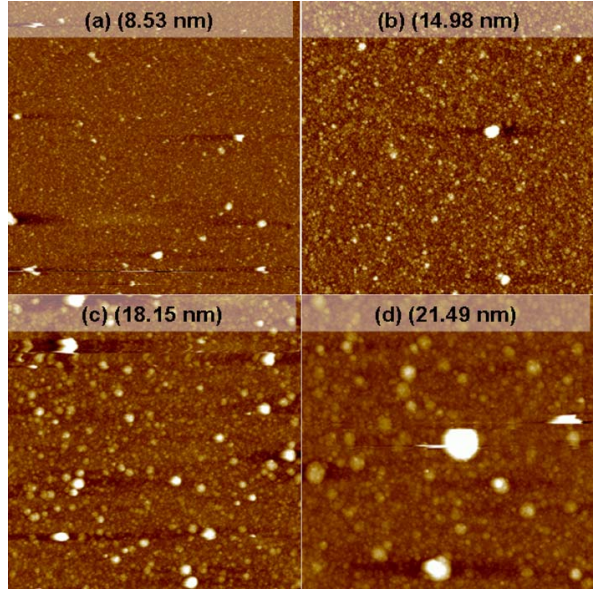

FIG. 6. (Color online) AFM images of 200 and 400 bilayers of ISAM films for deposition conditions (PAH-7/PCBS-7) $2 / 2 \min -15 \mathrm{~s}$ (a) for 200 bilayers and (b) for 400 bilayers; (PAH-9/PCBS-8) $2 / 2$ min-15 s (c) for 200 bilayers and (d) for 400 bilayers. The numbers in the parentheses are the root mean square roughness values. All images are on an area of $20 \times 20 \mu \mathrm{m}^{2}$.

heterogeneities that began to propagate during the LbL process at the higher bilayer numbers although the precise reason for this defect propagation is not known. This is consistent with the AFM images of the ISAM films taken at 200 and 400 bilayers for both (PAH-7/PCBS-7) $2 / 2$ min-15 s and (PAH-9/PCBS-8) $2 / 2$ min-15 s deposition conditions, as shown in Fig. 6. Films fabricated using the deposition condition (PAH-7/PCBS-7) $2 / 2$ min-15 s exhibited lower values of the root mean square (rms) surface roughness of approximately 8 and $15 \mathrm{~nm}$ for 200 and 400 bilayers, respectively, as compared to the films fabricated at (PAH-9/PCBS-8) $2 / 2$ min-15 s which had rms surface roughness values of approximately 18 and $21 \mathrm{~nm}$ for 200 and 400 bilayers, respectively.

To calculate the second order susceptibility, $\chi^{(2)}$, of the films, SHG measurements were taken. The square root of SHG is plotted versus the number of bilayers in Fig. 7. For the deposition condition (PAH-9/PCBS-8) $2 / 2$ min-15 s, a roughly linear relationship between square root of SHG and number of bilayers was observed for the first 200 bilayers implying that the polar orientation of the chromophores was maintained for the first 200 bilayers. The departure from linearity in Fig. 7 is likely related to the apparent decrease in the thickness per bilayer observed at 250 bilayers and higher.

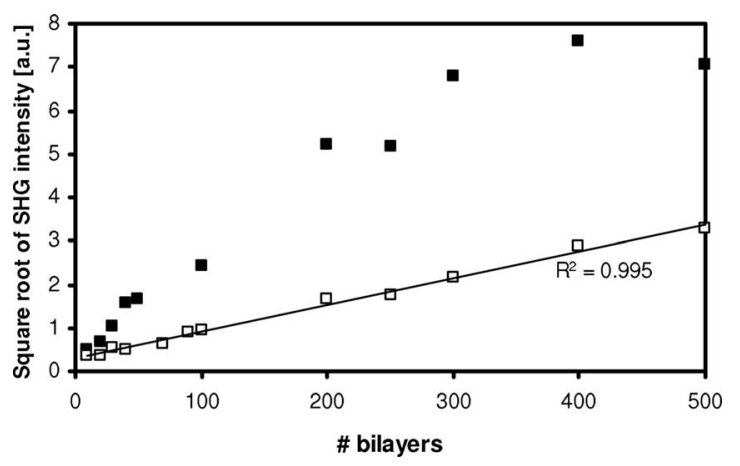

FIG. 7. Square root of SHG intensity vs the number of bilayers for (PAH-7/PCBS-7) $2 / 2$ min-15 s (open squares) and (PAH-9/PCBS-8) $2 / 2$ min-15 s (filled squares). 
TABLE III. Thickness, Absorbance per bilayer, tilt angle, and $\chi_{z z z}^{(2)}$ of the films fabricated at two different deposition conditions.

\begin{tabular}{|c|c|c|c|c|}
\hline Deposition condition & $\begin{array}{l}\text { Thickness per bilayer } \\
\text { (nm) }\end{array}$ & $\begin{array}{l}\text { Absorbance/bilayer } \\
\left(\times 10^{-3}\right)(\text { at } 450 \mathrm{~nm})\end{array}$ & $\begin{array}{c}\langle\bar{\psi}\rangle \\
(\mathrm{deg})\end{array}$ & $\begin{array}{c}\chi_{z z z}^{(2)} \\
\left(10^{-9} \text { esu }\right)\end{array}$ \\
\hline$(\mathrm{PAH}-7 / \mathrm{PCBS}-7)_{2 / 2} \min -15 \mathrm{~s}$ & $1.17( \pm 0.01)^{\mathrm{a}}$ & $0.9^{\mathrm{a}}$ & $37.3( \pm 2.2)^{\mathrm{a}}$ & $1.20( \pm 0.10)^{\mathrm{a}}$ \\
\hline$(\mathrm{PAH}-9 / \mathrm{PCBS}-8)_{2 / 2} \min -15 \mathrm{~s}$ & $2.75( \pm 0.09)$ (up to 250 bilayers) ${ }^{b}$ & $2.9^{\mathrm{b}}$ & $32.5( \pm 2.0)^{\mathrm{b}}$ & $2.24( \pm 0.16)$ (up to 200 bilayers $^{\mathrm{b}}$ \\
\hline
\end{tabular}

${ }^{\mathrm{a}}$ For $7 / 7 p \mathrm{H}$ conditions, the thickness/BL, absorbance/BL, tilt angle and SHG results were based on data up to 500 bilayers

${ }^{\mathrm{b}}$ For $9 / 8 p \mathrm{H}$ conditions, the data were reported for bilayer numbers as specified or up to 500 bilayers

Importantly, the square root of SHG versus number of bilayers for deposition condition (PAH-7/PCBS-7) $2 / 2$ min-15 s showed a linear relationship at least up to 500 bilayers; this means that the noncentrosymmetric polar ordering of the chromophores was maintained throughout the deposition process for this condition.

The film homogeneity was studied by taking three different measurements at different spots on the same sample. The standard deviation of the square root of SHG measurements for deposition conditions (PAH-7/PCBS-7) $2 / 2$ min-15 s and (PAH-9/PCBS-8) $2 / 2$ min-15 s are shown in Table II above for 200, 400, and 500 bilayers. For condition (PAH-9/PCBS-8) $2 / 2$ min-15 s, the standard deviation is comparatively higher for bilayer numbers 400 and 500 than for 200 bilayers and compared to deposition condition (PAH-7/PCBS-7) 2/2 min-15 s up to 500 bilayers. This higher standard deviation corresponds well with the visual appearance of these films which shows significant inhomogeneity.

Using Eqs. (2)-(5), $\chi_{z z z}^{(2)}$ and tilt angle were calculated and the results are summarized in Table III. Although the thickness per bilayer for deposition condition (PAH-9/PCBS-8) $2 / 2$ min-15 $\mathrm{s}$ is more than two times higher than that for (PAH-7/PCBS-7 $)_{2 / 2}$ min-15 s, which according to Eqs. (2) and (3) should lead to a lower value of $\chi_{\text {eff }}^{(2)}$ and hence a lower value of $\chi_{z z z}^{(2)}$, the slope of square root of SHG versus number of bilayers is almost three times higher for the elevated $p \mathrm{H}$ conditions than for the neutral $p \mathrm{H}$ conditions. This results in a higher value of $\chi_{z z z}^{(2)}$ for films deposited at higher $p \mathrm{H}$ than for the films deposited at neutral $p \mathrm{H}$. The absorbance per bilayer for films deposited at higher $p \mathrm{H}$ conditions is almost three times higher than those deposited at neutral $p \mathrm{H}$; this may imply a much higher concentration of chromophores at higher $p \mathrm{H}$ conditions. However, since absorbance of these films depends not only on the concentra-

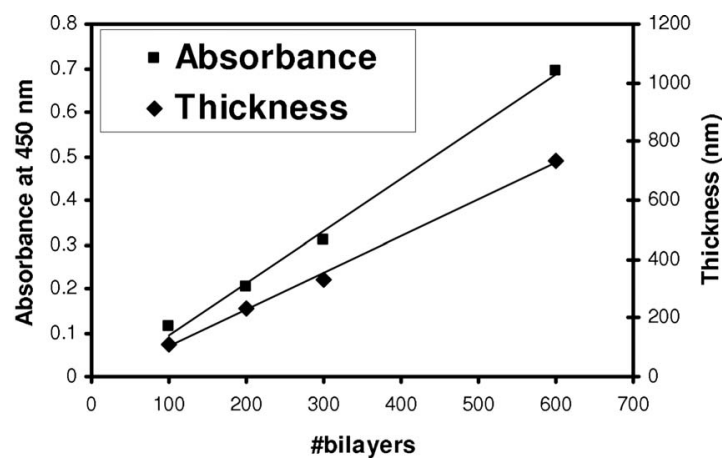

FIG. 8. Absorbance [squares] at $450 \mathrm{~nm}$ and thickness (diamonds) vs the number of bilayers for (PAH-7/PCBS-7) $2 / 2$ min for series made with an automated slide stainer. tion of the chromophores but also on the local chemical environment and the orientation of the chromophores, quantitative conclusions cannot be drawn about the chromophore concentration in these films. Since the effect of processing conditions on film properties is a central point of this paper, additional films were made with an automated slide stainer that employed somewhat different fabrication conditions. These are discussed next.

\section{Films made using an automated slide stainer}

In another set of experiments, LbL films were fabricated using an automated slide stainer. PAH and PCBS films up to 600 bilayers were deposited on glass slides. The polyelectrolyte solutions used were at $10 \mathrm{mM}$ concentration and at $p \mathrm{H}$ 7, conditions known from the previous work with the StratoSequence $^{\mathrm{TM}}$ Mark VI robotic deposition system to result in homogeneous films with long range polar order. The deposition and rinsing times were 2 min and this condition is written in abbreviated form as (PAH-7/PCBS-7 $)_{2 / 2 \mathrm{~min}}$. An absorbance per bilayer of 0.0012 at $450 \mathrm{~nm}$ was obtained using the spectrophotometer and a film thickness of 1.25 $( \pm 0.01) \mathrm{nm}$ per bilayer was estimated using the ellipsometer. These compare with an absorbance per bilayer of 0.0009 and a bilayer thickness of $1.17 \mathrm{~nm}$ for the films made with the StratoSequence instrument although the rinsing time for the latter films was $2 \mathrm{~min}, 15 \mathrm{~s}$. No convection was used in the deposition baths in contrast to the use of convection in the dipper used to fabricate films in Sec. III B. The absorbance and thickness data as a function of the number of bilayers are shown in Fig. 8.

As shown in Fig. 9, square root of SHG increased linearly with increasing number of bilayers indicating that the polar orientation of the films was maintained up to 600 bilayers and $740 \mathrm{~nm}$. Using the procedure described above, the tilt angle for these films was $38.0^{\circ}\left( \pm 2.4^{\circ}\right)$ and the value of

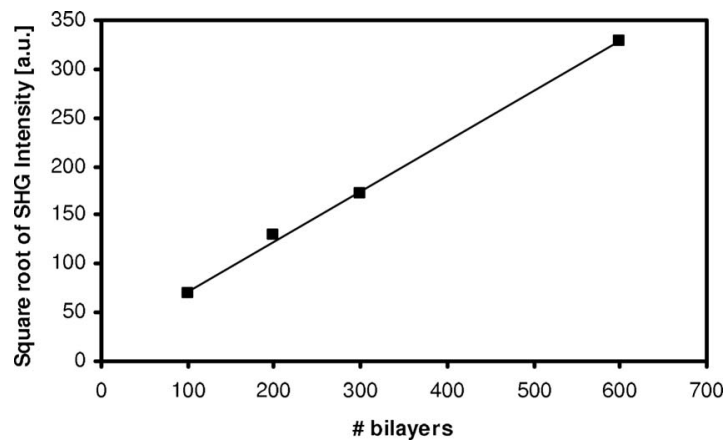

FIG. 9. Square root of SHG intensity vs the number of bilayers for (PAH-7/PCBS-7) $)_{2 / 2}$ min for series made with an automated slide stainer. 
$\chi_{z z z}^{(2)}$ was $1.25( \pm 0.005) \times 10^{-9}$ esu which is in excellent agreement with the values obtained in Sec. III B for the deposition condition (PAH-7/PCBS-7) $2 / 2$ min-15 s using the StratoSequence instrument reported in Table III.

\section{CONCLUSIONS}

Films containing an oriented, NLO chromophore were fabricated on glass slides using the ISAM process. The polymeric dye PCBS was deposited along with PAH while varying $p \mathrm{H}$ and deposition and rinsing times. It was observed that different deposition conditions strongly affected the optical properties of the ISAM films such as absorbance, thickness, and second order susceptibility $\chi_{z z z}^{(2)}$.

At deposition and rinsing times of 2 min, two series of ISAM films were fabricated at different $p \mathrm{H}$ conditions-one at $p \mathrm{H} 7$ for both polyelectrolytes and the other at $p \mathrm{H} 8$ for $\mathrm{PCBS}$ and $p \mathrm{H} 9$ for $\mathrm{PAH}$. Higher values of absorbance per bilayer and thickness per bilayer were observed for ISAM films when $\mathrm{PAH}$ was deposited at $p \mathrm{H} 9$ due to its reduced charge density which favors deposition with more loops and tails which would, in turn, lead to increased adsorption of PCBS. At the elevated $p \mathrm{H}$ condition $9 / 8$, the thickness per bilayer was found to be $2.7 \mathrm{~nm}$ as compared to $1.2 \mathrm{~nm}$ at the $p \mathrm{H}$ condition $7 / 7$. Considering that the deposition and rinsing times were the same, this implies that at higher $p \mathrm{H}$ the ISAM film formation process results in more rapid growth of the film to a given thickness. However, ISAM films made at higher $p \mathrm{H}$ did not show a linear growth in absorbance or thickness after 300 bilayers and exhibited visual inhomogeneity. By contrast, the films made at neutral $p \mathrm{H}$ exhibited linearity in absorbance, thickness, and square root of the SHG intensity as a function of the number of bilayers up to 500 bilayers. Furthermore, a second set of films made with a different automated deposition system at neutral $p \mathrm{H}$ also showed linearity of thickness and quadratic scaling of SHG intensity up to a thickness of $740 \mathrm{~nm}$.

These results demonstrate a window in processing conditions in which a trade-off between thickness per bilayer and optical homogeneity can be achieved by adjusting the $p \mathrm{H}$ of the polyelectrolyte solutions. The demonstration that ISAM films can be fabricated to thicknesses on the order of $1 \mu \mathrm{m}$ with a linear increase in thickness and uniform orientation of the chromophores establishes the possibility of fabricating EO waveguide devices with excellent thermal and temporal stability.

\section{ACKNOWLEDGMENTS}

The authors thank the following for financial support: NSF Grant No. ECS-0524625, the Macromolecules and Interfaces Institute at Virginia Tech for a fellowship for A.G., Luna Innovations (Subcontract No. 1194-RM-2S/VT), and the Institute for Critical Technology and Applied Science at
Virginia Tech. The authors also want to thank Steve McCartney for assistance with the AFM experiments.

${ }^{1}$ G. Decher and J. D. Hong, Makromol. Chem., Macromol. Symp. 46, 321 (1991).

${ }^{2}$ Nanoparticles and Nanostructured Films: Preparation, Characterization and Applications, edited by J. H. Fendler (Wiley, Weinhein, 1998).

${ }^{3}$ R. W. Boyd, Nonlinear Optics (Academic, New York, 1992).

${ }^{4}$ R. L. Sutherland, D. G. McLean, and S. Kirkpatrick, Handbook of Nonlinear Optics, 2nd ed. (Dekker, New York, 2003).

${ }^{5}$ L. R. Dalton, W. H. Steier, B. H. Robinson, C. Zhang, A. Ren, S. Garner, A. Chen, T. Londergan, L. Irwin, B. Carlson, L. Fifield, G. Phelan, C. Kincaid, J. Amenda, and A. Jend, J. Mater. Chem. 9, 1905 (1999).

${ }^{6}$ J. R. Heflin, C. Figura, and D. Marciu, Appl. Phys. Lett. 74, 495 (1999).

${ }^{7}$ M. J. Cho, S. K. Lee, J.-I. Jin, D. H. Choi, and L. R. Dalton, Thin Solid Films 515, 2303 (2006)

${ }^{8}$ H. Ma, S. Liu, J. Luo, S. Suresh, L. Liu, S. H. Kang, M. Haller, T. Sassa, L. R. Dalton, and A. K.-Y. Jen, Adv. Funct. Mater. 12, 565 (2002).

${ }^{9}$ D. Jungbauer, B. Reck, R. Twieg, D. Y. Yoon, C. G. Wilson, and J. D. Swalen, Appl. Phys. Lett. 56, 2610 (1990).

${ }^{10}$ J. W. Wu, J. F. Valley, S. Ermer, E. S. Binkley, J. T. Kenney, G. F. Lipscomb, and R. Lytel, Appl. Phys. Lett. 58, 225 (1991).

${ }^{11}$ Y. Shi, C. Zhang, H. Zhang, J. H. Bechtel, L. R. Dalton, B. H. Robinson, and W. H. Steier, Science 288, 119 (2000).

${ }^{12}$ L. R. Dalton, Pure Appl. Chem. 76, 1421 (2004).

${ }^{13}$ T.-D. Kim, J.-W. Kang, J. Luo, S.-H. Jang, J.-W. Ka, N. Tucker, J. B. Benedict, L. R. Dalton, T. Gray, R. M. Overney, D. H. Park, W. N. Herman, and A. K.-Y. Jen, J. Am. Chem. Soc. 129, 488 (2007).

${ }^{14}$ G. J. Ashwell, P. D. Jackson, and W. A. Crossland, Nature (London) 368, 438 (1994)

${ }^{15}$ P. Zhu, M. E. v. d. Boom, H. Kang, G. Evmenenko, P. Dutta, and T. J. Marks, Chem. Mater. 14, 4982 (2002).

${ }^{16}$ S. Yitzchaik, S. B. Roscoe, A. K. Kakkar, D. S. Allan, T. J. Marks, Z. Y. Xu, T. G. Zhang, W. P. Lin, and G. K. Wong, J. Phys. Chem. 97, 6958 (1993).

${ }^{17}$ E.-H. Kang, T. Bu, P. Jin, J. Sun, Y. Yang, and J. Shen, Langmuir 23, 7594 (2007).

${ }^{18}$ P. Fischer, M. Koetse, A. Laschewsky, E. Wisherhoff, L. Jullien, A. Persoons, and T. Berbiest, Macromolecules 33, 9471 (2000).

${ }^{19}$ A. Laschewsky, E. Wischerhoff, M. Kauranen, and A. Persoons, Macromolecules 30, 8304 (1997).

${ }^{20}$ Y. Lvov, S. Yamada, and T. Kunitake, Thin Solid Films 300, 107 (1997).

${ }^{21}$ M. J. Roberts, G. A. Lindsay, W. N. Herman, and K. J. Wynne, J. Am. Chem. Soc. 120, 11202 (1998).

${ }^{22}$ S. Dante, R. Advincula, C. W. Frank, and P. Stroeve, Langmuir 15, 193 (1999).

${ }^{23}$ X. Arys, A. Laschewsky, and A. M. Jonas, Macromolecules 34, 3318 (2001).

${ }^{24}$ C. Figura, P. J. Neyman, D. Marciu, C. Brands, M. A. Murray, S. Hair, R. M. Davis, M. B. Miller, and J. R. Heflin, Proc. SPIE 3939, 214 (2000).

${ }^{25}$ J. R. Heflin, M. T. Guzy, P. J. Neyman, K. J. Gaskins, C. Brands, Z. Wang, H. W. Gibson, R. M. Davis, and K. E. V. Cott, Langmuir 22, 5723 (2006).

${ }^{26}$ W. Kern, Semicond. Int. 7, 94 (1984)

${ }^{27}$ H. G. Tompkins and W. A. McGahan, Ellipsometry and Reflectometry (Wiley, New York, 1999).

${ }^{28}$ R. M. A. Azzam and N. M. Bashara, Ellipsometry and Polarized Light (North-Holland, Amsterdam, 1987).

${ }^{29}$ B. Schoeler, E. Poptoshev, and F. Caruso, Macromolecules 36, 5258 (2003).

${ }^{30}$ E. Poptoshev, B. Schoeler, and F. Caruso, Langmuir 20, 829 (2004).

${ }^{31}$ O. Mermut and C. J. Barrett, J. Phys. Chem. B 107, 2525 (2003).

${ }^{32}$ S. E. Burke and C. J. Barrett, Langmuir 19, 3297 (2003).

${ }^{33}$ K. Van Cott, M. Guzy, P. Neyman, C. Brands, J. Heflin, H. Gibson, and R. Davis, Angew. Chem., Int. Ed. 41, 3236 (2002).

${ }^{34}$ M. Fang, C. H. Kim, G. B. Saupe, H.-N. Kim, C. C. Waraksa, T. Miwa, A. Fujishima, and T. E. Mallouk, Chem. Mater. 11, 1526 (1999).

${ }^{35}$ A. Garg, J. R. Heflin, H. W. Gibson, and R. M. Davis, "Study of Film Structure and Adsorption Kinetics of Polyelectrolyte Multilayer Films: Effect of $\mathrm{pH}$ and Polymer Concentration," Langmuir (accepted).

${ }^{36}$ S. S. Shiratori and M. F. Rubner, Macromolecules 33, 4213 (2000). 